\title{
G (5 \\ Plasma Cell-free circRNAs Panel Act as Fingerprint Predicts the Occurrence of Laryngeal Squamous Cell Carcinoma
}

\section{Jiahui Han}

Department of Otolaryngology Head and Neck Surgery, The First People's Hospital of Lianyungang, The Affiliated Lianyungang Hospital of Xuzhou Medical University, Lianyungang, Jiangsu Province, 222061, PR China

\section{Qiuhong Lin}

Department of Otolaryngology Head and Neck Surgery, The First People's Hospital of Lianyungang, The Affiliated Lianyungang Hospital of Xuzhou Medical University, Lianyungang, Jiangsu Province, 222061, PR China

\section{Chunguang Dong ( $\nabla$ dongchunguang72@126.com )}

Department of Otolaryngology Head and Neck Surgery, The First People's Hospital of Lianyungang, The Affiliated Lianyungang Hospital of Xuzhou Medical University, Lianyungang, Jiangsu Province, 222061, PR China https://orcid.org/0000-0003-4576-2554

\section{Research}

Keywords: plasma, circRNA, LSCC, risk score function, ROC curve

Posted Date: February 8th, 2021

DOl: https://doi.org/10.21203/rs.3.rs-172078/v1

License: (c) (i) This work is licensed under a Creative Commons Attribution 4.0 International License. Read Full License 


\section{Abstract}

Background: Circular RNAs (circRNAs) have recently emerged as a new class of RNAs, highly enriched in the human tissues and very stable within cells, exosomes and body fluids. In this study, we aimed to screen the plasma cell-free derived circRNAs in laryngeal squamous cell carcinoma (LSCC) and investigate whether these circRNAs could predicted LSCC as potential biomarkers.

Methods: The circRNA microarray was employed with three samples in each group to screen the dysregulated circRNAs isolated from plasma samples. The top 20 circRNAs were first selected as candidates with the upregulated level in the plasma of LSCC. Results: Further validation found that only circ_0019201, circ_0011773 and circ_0122790 was consistent with training set. The ROC curve also revealed a high diagnostic ability an area under ROC curve value (AUC) for single circRNA and combined. The AUC for circ_0019201, circ_0011773 and circ_0122790 and the combined was 0.933, 0.908, 0.965 and 0.990 in training set. For the validation set, the AUC was $0.766,0.864,0.908$ and 0.951 . The three circRNAs were further investigated with stable expression in human plasma samples.

Conclusions: The plasma derived circ_0019201, circ_0011773 and circ_0122790 might be the potential biomarker for predicting the LSCC.

\section{Introduction}

Laryngeal squamous cell carcinoma (LSCC) is the most common tumor of the head and neck[1,2]. The main risk factors for laryngeal cancer include tobacco, human papillomavirus infection, laryngopharyngeal reflux, environmental and occupational exposure, and alcohol[3,4]. However, the molecular mechanism of the pathogenesis of LSCC remains unclear. In addition, although efforts have been made to develop an effective treatment for LSCC, the mortality rate for patients with LSCC remains high, with an overall 5-year survival rate of less than 50\%[5]. With the development of generation sequencing technology, many researchers have used omics data to predict the occurrence or prognosis of tumors. Studies have shown that the up-regulation and down-regulation of specific gene expression in serum may be associated with clear diagnosis and prognosis of tumor[6,7]. In clinical practice, squamous cell carcinoma of the neck is often diagnosed as other neck diseases, and in many cases, delayed diagnosis and treatment are inevitable. Therefore, it is of great clinical significance to develop a specific biomarker for cervical squamous cell carcinoma.

With the development of high-throughput sequencing technology, more and more non-coding RNA (ncRNA) was found in human genome. Accumulating evidence have demonstrated that these ncRNAs have a series of crucial regulatory potentials both in transcription and post-transcription, which participated in many biological processes[8]. Circular RNAs (circRNAs) are important members of the non-coding RNA family, and they are single-stranded closed circular RNA molecules without a 5'-end cap or a 3 '-end poly $(A)$ tail, which are formed by covalent bonding $[9,10]$. Furthermore, researchers have indicated the differential circRNA profiles of the serum exosomes derived from patients with other human 
malignant tumors, such as gastric cancer, colon cancer and hepatocellular carcinoma[11,12]. However, no evidence was found regarding the circRNA in LSCC patients.

In this study, we investigated the potential use of circulating cell-free circRNAs in plasma as biomarkers for LSCC. First, we integrated abnormal/deregulated circRNAs by analyzing the microarray data from the plasma samples of LSCC patients and non-cancer controls. The top 20 dysregulated circRNAs were then confirmed and validated by reverse transcriptional quantitative polymerase chain reaction (RT-qPCR) assay. A multi-stage validation, including a training set and a validation set, was then performed to test whether abnormally expressed plasma derived circRNAs which might predict LSCC in healthy volunteers.

\section{Methods}

\section{Study design}

A retrospective case-control study was conducted at the First People's Hospital of Lianyungang. A total of 120 patients with LSCC who were diagnosed with LSCC in department of Otolaryngology Head and Neck Surgery of the First People's Hospital of Lianyungang was enrolled from January 1, 2015, to July 1, 2019. This study was approved by an institutional review board of The First People's Hospital of Lianyungang. Written informed consents were obtained from all enrolled subjects. Peripheral blood samples of patients were collected before surgery or chemoradiotherapy. Blood samples were collected in a separate vacuum cube, followed by centrifugation at $3,000 \mathrm{rpm}$ for $10 \mathrm{~min}$. All samples were stored at $-80^{\circ} \mathrm{C}$ until further analysis. The relevant clinical data of all patients were available. None of these patients had received any treatment such as radiotherapy or chemotherapy prior to surgery. The initial treatment was surgery. The diagnosis of LSCC was confirmed by the histopathological analysis of tumor tissue obtained after surgical resection. Ten ${ }^{\text {th }}$ edition of American Joint Committee on Cancer (AJCC) tumor-node-metastasis (TNM) systems was used while staging the disease based on clinical, radiological, and pathological findings. The detailed information of patients and healthy controls was summarized in Table1.

\section{CircRNA microarray and analysis}

RNA was extracted from three plasma samples diagnosed with LSCC and three healthy controls as circulating samples, both of which were used for microarray detection. Each sample was tested with a 1.0 $\mu \mathrm{g}$ total RNA. The microarray was detected by using Human CircRNA Microarray V2 (CapitalBio, Beijing, China). We screened out all the low-expression genes before performing other analyses. We only retained genes with at least half markers in five out of samples. This reduces the initial 37,681 input genes in each sample to about 15,000 detected genes. In the following analysis, we used this set of expression filter genes. We performed a bilateral Mann-Whitney $U$ test performed by Wilcox. Finally, we use the BenjaminHochberg correction (R. P. Adojust function) to explain the multiple tests. For the purpose of analysis, we found that the genes significantly differentially expressed were those with a Benjamin-Hochberg $Q$ value $<0.05$. We also used the Limma package to perform parallel differential expression analysis. All further data analysis and visualization is performed using custom $\mathrm{R}$ scripts. 
Total RNA was extracted using TRIzol Reagent (Invitrogen, CA, USA) according to the manufacturer's instructions. RNA quantity and quality were measured with a Nano Drop ND-1000 spectrophotometer (Thermo, USA). RNA integrity was also assessed by standard denaturing $1 \%$ agarose gel electrophoresis. The synthetic C. elegans miRNA (cel-miR-39, Applied Biosystems, Foster City, CA, USA) was added to each sample as external reference. The cDNA was generated by using the Super-Script First-Strand Synthesis System (Invitrogen, Carlsbad, CA, USA). Transcript levels were measured in duplicate by qPCR (ABI 7900; Life Technologies; Thermo Fisher Scientific, Inc.). The amplification procedure was $95^{\circ} \mathrm{C}$ for predenaturation for $30 \mathrm{sec} ; 95^{\circ} \mathrm{C}$ for $5 \mathrm{sec}, 60^{\circ} \mathrm{C}$ for $31 \mathrm{sec}\left(45 \mathrm{cycles}\right.$ ); dissolution curves $95^{\circ} \mathrm{C}$ for $15 \mathrm{sec}$, $60^{\circ} \mathrm{C}$ for $60 \mathrm{sec}, 95^{\circ} \mathrm{C} 15 \mathrm{sec}$. The relative expression of circRNA was normalized to GAPDH and cel-miR39 and was calculated using the $2^{-\triangle \Delta C q}$ method.

\section{Screening phase}

The screening phase was divided into training set and validation set. 20 samples each group were enrolled while the validation set contained another 100 samples in each group. Groups were named as healthy volunteers and LSCC patients.

Risk score was applied to analyze the prediction ability for certain circRNAs. In brief, the upper $95 \%$ reference interval $(95 \% \mathrm{Cl})$ of each circRNA value in the controls group was used as the cutoff for the expression level of the certain circRNA. If the expression of circRNA in this sample was higher than the $95 \% \mathrm{Cl}$, we assigned as 1 , if lower, we assigned as 0 . The risk score was defined according to a linear combination of the expression level for each circRNA. For example, the RSF for sample i using information from four circRNAs was: $r s f i=\sum 3 j-1$ Wj.sij. In the above equation, sij is the risk score for circRNA $j$ on sample $i$, and $W j$ is the weight of the risk score of circRNA $j$. The risk score of four circRNAs was calculated using the weight by the regression coefficient that was derived from the univariate logistic regression analysis of each circRNAs. Samples were ranked according to their RSF and then divided into a high-risk group, representing the LSCC group, and a low-risk group, representing the predicted control individuals.

\section{Statistical analysis}

If no special, data was presented as Mean $\pm S E M$. The classification variables were counted by chi-square test. Continuous variables were counted by the student's $t$ test. Statistical analysis was performed using STATA 10.0, and presented with GraphPad Prism 5.0. Results were considered statistically significant at $P$ $<0.05$.

\section{Results}

The circRNA expression profile in LSCC and control group. 
The circRNA microarray was further employed. Each group contains three samples. Hierarchical clustering analysis and volcano plot distribution were used to sort the aberrantly expressed circRNAs in different groups. As presented in Fig. 1a, we obtained a different expression level of circRNA in each group. We used the following parameters for further screening: i, P value $<0.05$; ii, CT value $<35$; iii, detection rate $>75 \%$. A total of 122 circRNA transcripts were specifically increased in LSCC group comparing with control group while 98 circRNAs was proved with downregulated (Fig. 1b). In order to reveal the potential biomarker for LSCC, we selected the top 20 increased circRNAs in LSCC group as candidate diagnostic makers (Fig. 1c).

\section{Validation of significantly dysregulated circRNAs in plasma}

Two staged validation of candidate circRNAs was used including 20 paired samples as training set and 100 paired samples as validation set. A randomly selected 20 paired LSCC and control samples was used. The top 20 circRNAs was first validated. Among the 20 candidate circRNAs, 10 circRNAs was confirmed with significant different expression; however, 5 circRNAs was consistent with microarray results while the rest circRNAs was presented with decreased level in LSCC group.

Based on the results in training set, the rest 100 paired samples were enrolled as validation set. We next examine the expression of the five candidate circRNAs in validation set. We found that hsa_circ_0055202, hsa_circ_0074920 and hsa_circ_0043722 was confirmed with higher expression level in LSCC, while hsa_circ_0010178 presented no significant. The hsa_circ_0009760 presented a higher expression in IncRLSCC; however, the $p$ value was $<0.05$.

Two-stage validation of candidate circRNAs was performed, with 20 pairs of paired samples as the training set and 100 pairs as the validation set. As shown in Fig. 2a, 20 pairs of LSCC and control samples were randomly selected. The top 20 circRNAs were identified for the first time. Among the 20 candidate circRNAs, three circRNAs entitled circ_0019201, circ_0011773 and circ_0122790 were proved to be differentially expressed and consistent with the microarray results. However, another four circRNAs were inconsistent with the microarray results, and the remaining circRNAs presented no difference between LSCC group and control group (Fig. 2b).

Based on the results of the training set, the remaining 100 paired samples were enrolled in the verification set. We then studied the expression of the circRNAs validation set of the three candidates. As shown in Fig. 3, we found that circ_0019201, circ_0011773 and circ_0122790 had higher expression levels confirmed by LSCC. Furthermore, the expression of the three circRNA presented a remarkable decreasing level after surgical excision.

\section{Risk score analysis}

In order to further explore the accuracy and specificity of these three circRNAs as potential signatures, risk scoring formulas were used to evaluate the diagnostic value of the 3 circRNAs. Firstly, we divided the control and case groups in the training set according to the upper $95 \%$ confidence interval $(95 \% \mathrm{Cl})$ of the 
control group. Logistic regression analysis was used to calculate the risk score. All plasma samples were then divided into a high-risk group (possibly LSCC) and a low-risk group (predicted to be a control group).

We define the cutoff value as the maximum of sensitivity + specificity. The positive predictive value (PPV) and negative predictive value (NPV) calculated in the training set were $90 \%$ and $85 \%$ respectively. We further applied the same values to calculate the risk score for the validation set sample, with PPV and NPV of $90 \%$ and $89 \%$, respectively (Table 2). In addition, we also used ROC curve analysis to evaluate the predictive diagnostic value of circRNA for LSCC. In the test set, the areas under the ROC curve of the three circRNAs were $0.933,0.908$ and 0.965 , respectively, and the combination of the three had a good ability to distinguish the areas under the ROC curve of the LSCC patients from the control group. In the validation set of enlarged samples, the areas under the ROC curve of the three circRNAs were 0.766, 0.864 and 0.908 , respectively. Combined with the three circRNAs, the area under the ROC curve of the LSCC patients and the control group was 0.951 (Fig. 4).

\section{Stability detection of circRNAs in plasma samples}

We next amplified the three circRNAs in five healthy controls. We incubated human plasma obtained from three healthy controls at room temperature for $0 \mathrm{~h}, 12 \mathrm{~h}$ and $24 \mathrm{~h}$, treated with frozen thawing for 5 cycles, under storage of $-80^{\circ} \mathrm{C}$ for about 7 days and digesting with RNAse. We found that neither the expression level of the three circRNAs were alternated indicated that circ_0019201, circ_0011773 and circ_0122790 were stably expressed and detectable in human plasma (Fig. 5).

\section{Discussion}

In the past decade, great progress has been made in the research and treatment of LSCC[13,14]. However, treatment resistance increases the recurrence rate of LSCC and patients who undergo total laryngectomy have a poor quality of life[15]. This study screened the differentially expressed genes to provide ideas for subsequent studies, including the exploration of potential diagnostic targets, which is conducive to the risk assessment of LSCC at an earlier stage.

Previous published studies on biomarkers of LSCC were mainly focused on proteins and miRNAs or IncRNAs. For example, some researchers have found that HOTAIR, a long non-coding RNA, was significantly increased in LSCC and is closely related to tumor progression, as well as the occurrence, metastasis, and poor prognosis of tumors[16]. In addition, abnormally elevated MALAT1 expression was also found in LSCC tumor tissues, suggesting a poor prognosis for patients[17]. The long non-coding RNA UCA1 was also highly expressed in LSCC[18]. However, the current studies on non-coding RNA in LSCC mainly focus on tumor tissues and tumor cell models, and there were still few studies on the exploration of LSCC-related diagnostic markers in cell-free plasma samples. Therefore, starting from the plasma samples of LSCC patients, this study focused on cell-free circRNAs in plasma samples by using high-throughput microarray technology. Based on the stability of circRNA expression, the potential of circRNA as a disease risk prediction target was further explored, and the study was innovative to a certain extent. 
Circulating non-coding RNAs have recently emerged as novel biomarkers for cancer development and progression. Among the studies on non-coding RNAs as diagnostic markers, miRNAs were the first to be reported, and there have been commercialized kits for the diagnosis of disease models, followed by long non-coding RNAs. However, with the deepening understanding of non-coding RNA, more and more attention has been paid to the clinical value of circRNAs in tumor diagnosis. Xu et al. identified circRNA_0000826 as a potential diagnostic biomarker for liver metastasis from colorectal cancer through RNA sequencing[19]. In addition, Tang et al. also found that gastric cancer-tissue derived circ-KIAA1244 could be used as a novel circulating biomarker for gastric cancer detection[11]. These results all proved the feasibility of peripheral circulating circRNA as a future diagnostic target in LSCC.

\section{Conclusions}

In summary, in this study, we used a microarray-based approach to screen the potential fingerprints of LSCC. we found that the circ_0019201, circ_0011773 and circ_0122790 Panel may be able to predict LSCC in the normal population with relatively high sensitivity and specificity, suggesting that circ_0019201, circ_0011773 and circ_0122790 may be used as an early disease prediction model for LSCC in the future. However, due to the limitation of the sample size in this study, more samples are needed for verification, and further studies are needed to confirm the potential regulatory mechanism of these circRNAs in the development of LSCC.

\section{Abbreviations}

LSCC, laryngeal squamous cell carcinoma; circRNA, circular RNA; qRT-PCR, quantitative real-time polymerase chain reaction; ROC curve: Receiver Operating Characteristic curve; AUC: Area under the Curve; RSF: Risk score function

\section{Declarations}

\section{Acknowledgements}

Not applicable.

\section{Authors' contributions}

$\mathrm{JHH}$ and QHL: manuscript writing, literature search and data analysis; $\mathrm{JHH}$ : data analysis and statistical analysis. CGD: research design. All authors read and approved the final manuscript.

\section{Funding}

No funding received.

\section{Availability of data and materials}


The datasets used and/or analyzed during the current study are available from the corresponding author on reasonable request.

\section{Ethics approval and consent to participate}

Ethical approval was obtained from the Ethics Committee of the First People's Hospital of Lianyungang. All procedures performed in studies involving human participants were in accordance with the ethical standards of the institutional and national research committee. Informed consent was obtained from all individual participants included in the study.

\section{Consent for publication}

Not applicable.

\section{Competing interests}

The authors declare that they have no competing interests.

\section{References}

1. Li B, Li B, Jiang H, Yang Y, Zhang X, Su Y, Hua R, Gu H, Guo X, Ye B, Yang Y, He Y, Sun Y, Piessen G, Hochwald SN, Cuesta MA, Birdas TJ, Li Z, written on behalf of the AMETSCG. The value of enhanced CT scanning for predicting lymph node metastasis along the right recurrent laryngeal nerve in esophageal squamous cell carcinoma. Annals of translational medicine. 2020; 8: 1632.

2. Li Y, Wu T, Gong S, Zhou H, Yu L, Liang M, Shi R, Wu Z, Zhang J, Li S. Analysis of the Prognosis and Therapeutic Value of the CXC Chemokine Family in Head and Neck Squamous Cell Carcinoma. Frontiers in oncology. 2020; 10: 570736.

3. Jin Z, Zhang B, Zhang L, Huang W, Mo X, Chen Q, Wang F, Chen Z, Li M, Zhang S. Immune-checkpoint inhibitor plus chemotherapy versus conventional chemotherapy for treatment of recurrent or metastatic head and neck squamous cell carcinoma: a systematic review and network metaanalysis. Therapeutic advances in medical oncology. 2020; 12: 1758835920983717.

4. Korpela SP, Hinz TK, Oweida A, Kim J, Calhoun J, Ferris R, Nemenoff RA, Karam SD, Clambey ET, Heasley LE. Role of epidermal growth factor receptor inhibitor-induced interferon pathway signaling in the head and neck squamous cell carcinoma therapeutic response. Journal of translational medicine. 2021; 19: 43.

5. Wang X, Guo J, Yu P, Guo L, Mao X, Wang J, Miao S, Sun J. The roles of extracellular vesicles in the development, microenvironment, anticancer drug resistance, and therapy of head and neck squamous cell carcinoma. Journal of experimental \& clinical cancer research : CR. 2021; 40: 35.

6. Xia Y, Wang Q, Huang X, Yin X, Song J, Ke Z, Duan X. miRNA-Based Feature Classifier Is Associated with Tumor Mutational Burden in Head and Neck Squamous Cell Carcinoma. BioMed research international. 2020; 2020: 1686480. 
7. Muzaffar J, Bari S, Kirtane K, Chung CH. Recent Advances and Future Directions in Clinical Management of Head and Neck Squamous Cell Carcinoma. Cancers. 2021; 13.

8. Wei WF, Zhou CF, Wu XG, He LN, Wu LF, Chen XJ, Yan RM, Zhong M, Yu YH, Liang L, Wang W. MicroRNA-221-3p, a TWIST2 target, promotes cervical cancer metastasis by directly targeting THBS2. Cell death \& disease. 2017; 8: 3220.

9. Chen W, Quan Y, Fan S, Wang H, Liang J, Huang L, Chen L, Liu Q, He P, Ye Y. Exosome-transmitted circular RNA hsa_circ_0051443 suppresses hepatocellular carcinoma progression. Cancer letters. 2020; 475: 119-28.

10. Luo Z, Rong Z, Zhang J, Zhu Z, Yu Z, Li T, Fu Z, Qiu Z, Huang C. Circular RNA circCCDC9 acts as a miR-6792-3p sponge to suppress the progression of gastric cancer through regulating CAV1 expression. Molecular cancer. 2020; 19: 86.

11. Tang W, Fu K, Sun H, Rong D, Wang H, Cao H. CircRNA microarray profiling identifies a novel circulating biomarker for detection of gastric cancer. Molecular cancer. 2018; 17: 137.

12. Zhuo F, Lin H, Chen Z, Huang Z, Hu J. The expression profile and clinical significance of circRNA0003906 in colorectal cancer. OncoTargets and therapy. 2017; 10: 5187-93.

13. Zhu Z, Liu Y, Wu D, Wang H. Association Between Mitochondrial DNA Copy Number and Head and Neck Squamous Cell Carcinoma: A Systematic Review and Dose-Response Meta-Analysis. Medical science monitor : international medical journal of experimental and clinical research. 2021; 27: e928327.

14. Romani C, Salviato E, Paderno A, Zanotti L, Ravaggi A, Deganello A, Berretti G, Gualtieri T, Marchini S, D'Incalci M, Mattavelli D, Piazza C, Bossi P, Romualdi C, Nicolai P, Bignotti E. Genome-wide study of salivary miRNAs identifies miR-423-5p as promising diagnostic and prognostic biomarker in oral squamous cell carcinoma. Theranostics. 2021; 11: 2987-99.

15. Wu ZH, Tang Y, Zhou Y. A Metabolic Gene Signature to Predict Overall Survival in Head and Neck Squamous Cell Carcinoma. Mediators of inflammation. 2020; 2020: 6716908.

16. Li T, Qin Y, Zhen Z, Shen H, Cong T, Schiferle E, Xiao S. Long non-coding RNA HOTAIR/microRNA-206 sponge regulates STC2 and further influences cell biological functions in head and neck squamous cell carcinoma. Cell proliferation. 2019; 52: e12651.

17. Liu X, Zhao W, Wang X. Inhibition of long non-coding RNA MALAT1 elevates microRNA-429 to suppress the progression of hypopharyngeal squamous cell carcinoma by reducing ZEB1. Life sciences. 2020; 262: 118480.

18. Fang Z, Zhao J, Xie W, Sun Q, Wang H, Qiao B. LncRNA UCA1 promotes proliferation and cisplatin resistance of oral squamous cell carcinoma by sunppressing miR-184 expression. Cancer medicine. 2017; 6: 2897-908.

19. Shi L, Tao C, Tang Y, Xia Y, Li X, Wang X. Hypoxia-induced hsa_circ_0000826 is linked to liver metastasis of colorectal cancer. Journal of clinical laboratory analysis. 2020; 34: e23405.

\section{Tables}


Table1. Clinicopathological characteristics of LSCC and cancer-free control samples

\begin{tabular}{|llll|}
\hline Characteristics & LSCC & Control & Pvalue \\
\hline N & 120 & 120 & \\
\hline Age Mean (SE) year & $55.12(4.31)$ & $56.25(5.77)$ & $0.21^{\mathrm{a}}$ \\
\hline Gender (male/female) & $79 / 41$ & $82 / 38$ & $0.68^{\mathrm{b}}$ \\
\hline Location & & & \\
\hline Lower & 21 & & \\
\hline Middle & 68 & & \\
\hline Upper & 31 & \\
\hline Tumor size & & \\
\hline$<4 \mathrm{~cm}$ & 55 & \\
\hline 4cm & 65 & \\
\hline TNM stage(l::: $\mathbb{\text { X) }}$ & $62: 33: 25$ & \\
\hline Lymph node metastasis & & \\
\hline Yes & 89 & \\
\hline No & 31 & \\
\hline
\end{tabular}

a Student t-test.

${ }^{b}$ Chi-square test.

Table 2. Risk score analysis of in LSCC and cancer-free control plasma samples

\begin{tabular}{|lcccc|}
\hline Score & $\mathbf{0 - 7 . 0 8}$ & $\mathbf{7 . 0 8 - 1 1 . 0 2}$ & PPV $^{\mathrm{a}}$ & NPV $^{\mathrm{b}}$ \\
\hline Training set & & & 0.90 & 0.85 \\
\hline LSCC & 2 & 18 & & \\
\hline Control & 17 & 3 & & \\
\hline Validation set & & & 0.90 & 0.89 \\
\hline LSCC & 10 & 90 & & \\
\hline Control & 89 & 11 & & \\
\hline
\end{tabular}


aPPV, positive predictive value.

bNPV, negative predictive value.

\section{Figures}

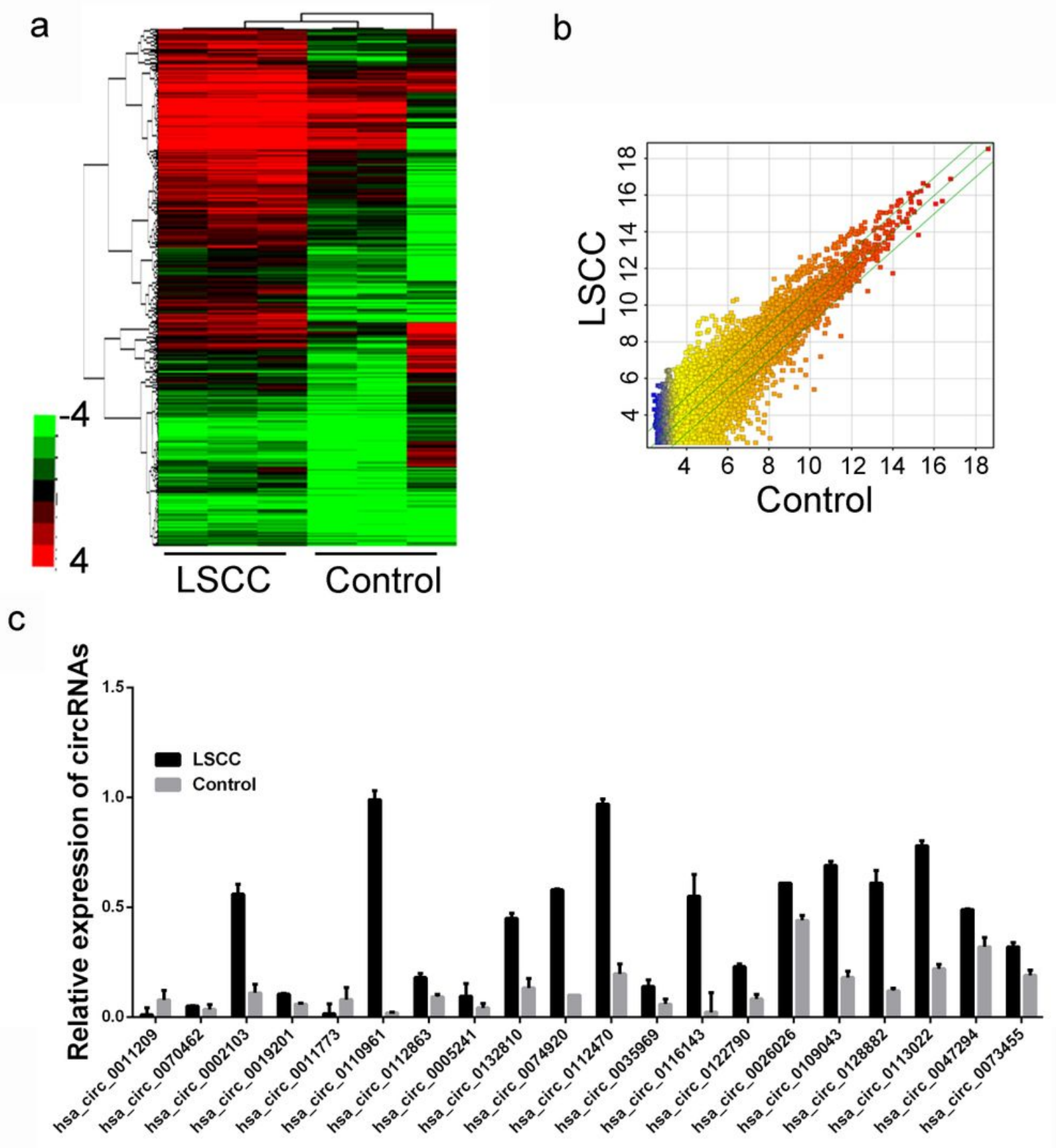

Figure 1 
circRNA expression profiling in circulating samples and tissues samples. A: Cluster analysis and scatter plot of the different expressed circRNAs (Three plasma samples from patients diagnosed with LSCC and three cancer-free controls). B: Rhe scatter plots of dysregulated circRNA, $p<0.05$. C:The top upregulated circRNAs in LSCC group through microarray detection.

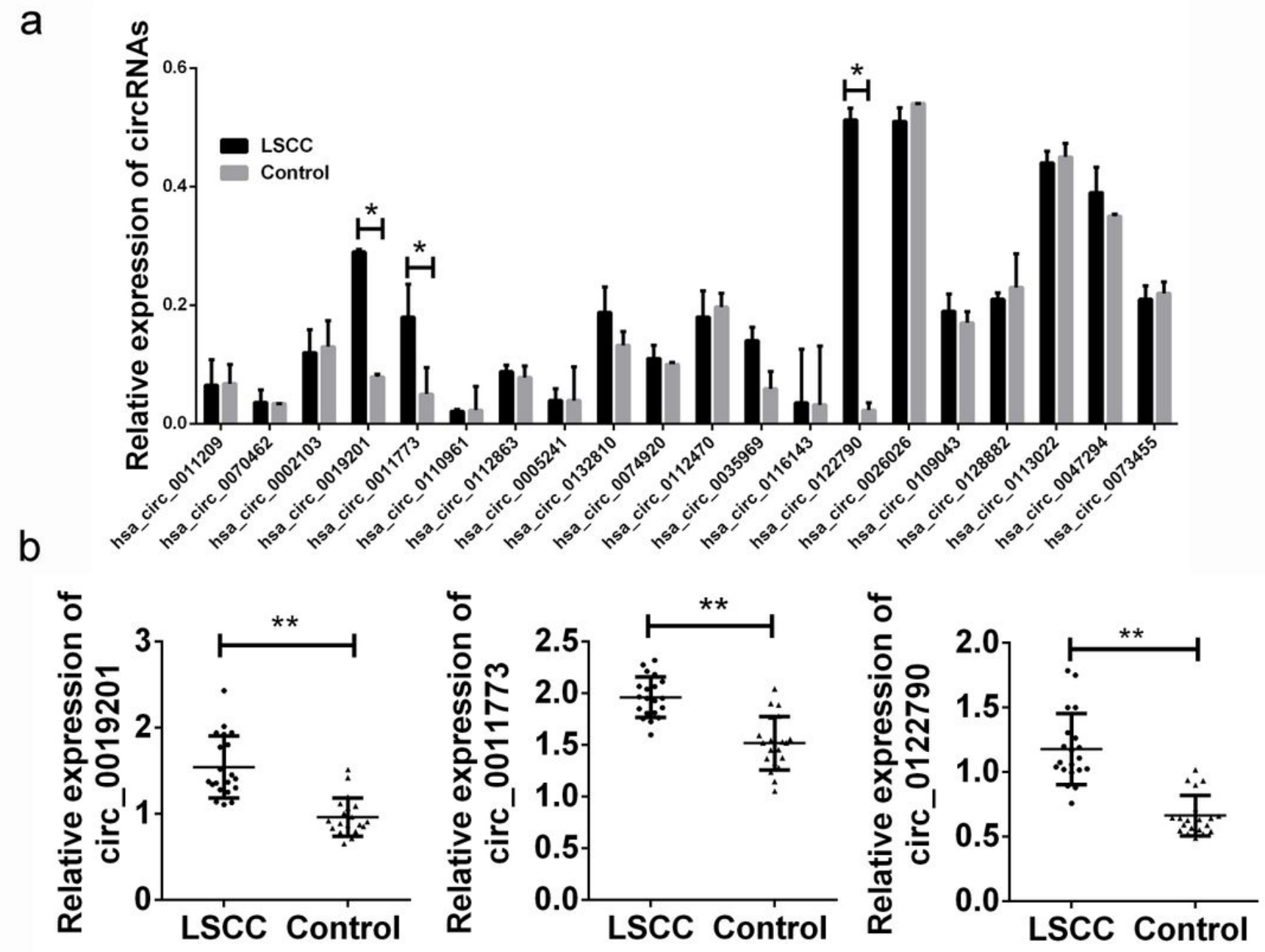

Figure 2

Validation of candidate circRNA in training set. A: Total 20 paired plasma from LSCC patients, and 20 cancer-free controls were used in RT-qPCR analysis. B: The detailed expression of circ_0019201, circ_0011773 and circ_0122790 in LSCC and control groups. Data was presented as mean \pm SEM. Data was analyzed with student $t$ test. n.s. indicated no significant, * indicated $p<0.05$ and $* \star$ indicated $p<$ 0.01 . 

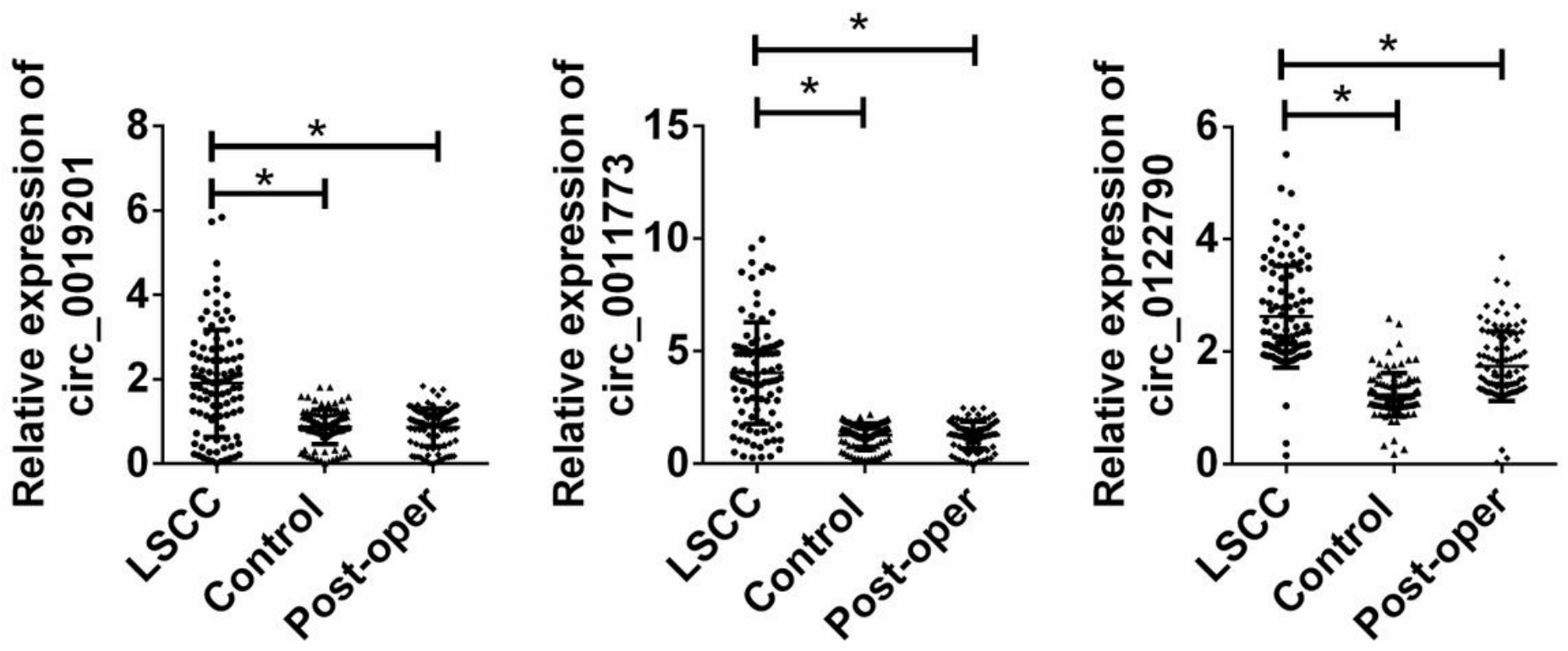

Figure 3

Validation of candidate circRNA in $\mathrm{n}$ validation set Total 100 paired plasma from LSCC patients, and 100 cancer-free controls were used in RT-qPCR analysis. Data was presented as mean \pm SEM. Data was analyzed with student $t$ test, * indicated $p<0.05$.
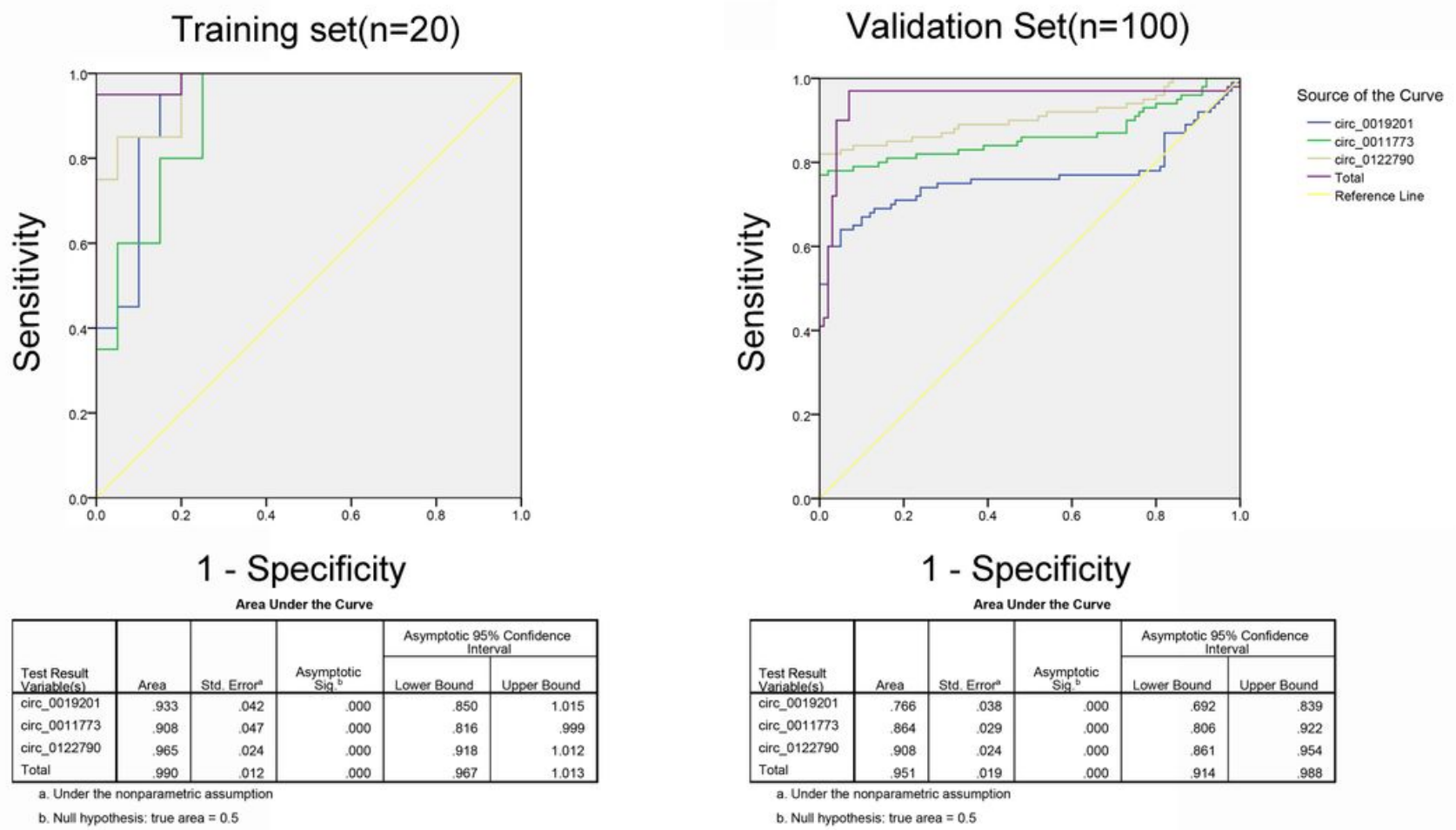
Figure 4

ROC analysis of the three potential biomarkers for LSCC by using risk score analysis.
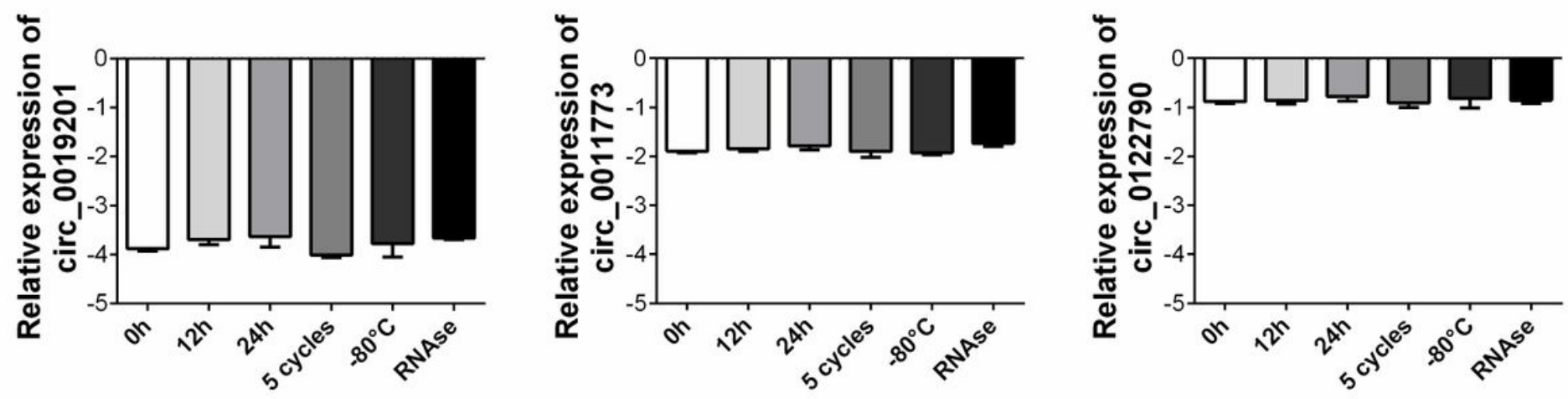

Figure 5

Stability detection of the three circRNAs in human plasma RT-qPCR was applied for detecting the expression level of the three circRNAs. Data was presented as mean \pm SEM with log-transformed. No significant difference was observed in each group. 